\title{
Open Amputation in Preterm Neonatal Digits With Post-Operative Management Using Medical Grade Honey
}

\section{Eleftherios Smaropoulos*, Eleni Papakrivou, Dimitrios Netskos, Odysseas Gkikas}

Neonatal Intensive Care Unit, Iaso General Hospital, Larisa, Greece

\section{Abstract}

Cutaneous development is highly dependent on gestational maturity, making wound management difficult and critical to the well being and survival of neonates. This case demonstrates the successful use of a medical grade honey product in the management of open amputation wounds in a preterm 28 -week gestation neonate.

Taking into account the known benefits of honey in wound care and applying them in this scenario, post-operative use of the medical grade honey product demonstrated rapid wound healing with once daily application for 15 days. No side effects were observed, with minimal to no scarring. Upon follow-up at 7 months the results remained esthetically acceptable.

Lack of research regarding wounds and their respective management in neonates and infants makes it difficult to establish standards of care. Continued studies are necessary so that NICUs may be better prepared to successfully receive these fragile patients. Medical grade honey may hold the potential to be incorporated into wound management protocols throughout hospitals around the world.

\section{Publication History:}

Received: May 26, 2017

Accepted: June 28, 2017

Published: June 30, 2017

\section{Keywords:}

Neonatology, Pediatrics,

Prematurity, Wound care, Wound management

\section{Product Notation:}

${ }^{\star}$ L-Mesitran ${ }^{\circledR}$ Ointment, Triticum, Maastricht, Netherlands

\section{Introduction}

After birth the skin transitions from an in utero environment containing amniotic fluid into a comparatively dry and gaseous microbe-rich environment [1,2]. With cutaneous function not fully developed in neonates, the risks of complications become much higher. Therefore, during the first weeks of life many factors need to be considered, such as risk of infection, skin breakdown, thermoregulation and absorption of topical agents [2].

Closely related to the lack of skin integrity is the high incidence of extravasation injuries in very preterm infants. This is even higher due to their dependence on intravenous fluids for survival [3]. Such occurrences become an enormous challenge in hospitalised neonates, often due to consequent skin necrosis and potential functional and esthetic impairment [4].

The use of honey in medicine dates back to ancient times and is undergoing resurgence in modern medicine. It is known to display both antimicrobial and wound healing properties, with no reported resistance $[5,6]$. With the advances in wound care and the availability of medical grade honey, its use is increasingly documented in adult patients but still moves at a noticeably slower pace in pediatrics and neonatology [6,7]. This case demonstrates a rare clinical scenario in the field of neonatal care, which benefited from post-operative management with a medical grade honey product (L-Mesitran ${ }^{\circ}$ Ointment, Triticum, Maastricht, Netherlands).

\section{Case Presentation}

A preterm 28-week gestation male neonate of IVF pregnancy was admitted with respiratory deficiency at the Neonatal Intensive Care Unit (NICU) of the Iaso General Hospital, Larisa, Greece. As an inpatient he was submitted to vascular line placement in a vein of the middle axillary area and treated with TPN and medication (fentanyl, dopamine, ampicillin, gentamycin, ceftriaxone and caffeine), for seventy two hours.

On the third day of hospitalization an extravazation injury occured, leading to arterial thrombosis of the right hand digital arteries. Despite the use of warm dressings, massages and vasodilatory creams such as topical nitroglycerine ointment, as well as allopurinol to prevent further ischemia, arterial occlusion led to permanent digit compromise. Heparin was not used due to the risk of intracranial hemorrhage.

Dry gangrene presented on digits two through five, three days after initial presentation of the injury (Figure 1). Conservative treatment with allopurinol and moisturising and vasodilatory agents as described, was insufficient to prevent open amputation of the involved digits, performed at day eight of life (Figure 2). With the intent to save as much tissue as possible, no dermal flaps or bioengineered skin substitutes were used to cover bone areas.

Following surgery post-operative management was initiated with a medical grade honey product (L-Mesitran ${ }^{\circ}$ Ointment, Triticum, Maastricht, Netherlands), which was applied once daily, for fifteen days. Rapid onset of the healing process occurred, with the beginning of epithelialization visible at day three, progressing to near completion at day ten and being totally complete at day fifteen, therefore ending treatment.

Upon follow-up at seven months of age, the esthetic results were fully acceptable, with minimal to no visible scarring (Figure 3 ).

\section{Discussion}

With structural integrity of the stratum corneum (SC) being related to gestational age at birth, the premature infant presents a poor epidermal barrier, with fewer cornified layers than when compared to

"Corresponding Author: Dr. Eleftherios Smaropoulos, Neonatal Intensive Care Unit, laso General Hospital, Larisa, Greece; E-mail: Ismaropoulos@gmail.com

Citation: Smaropoulos E, Papakrivou E, Netskos D, Gkikas O (2017) Open Amputation in Preterm Neonatal Digits With Post-Operative Management Using Medical Grade Honey. Int J Pediatr Neonat Care 3: 125. doi: https://doi. org/10.15344/2455-2364/2017/125

Copyright: (C) 2016 Smaropoulos et al. This is an open-access article distributed under the terms of the Creative Commons Attribution License, which permits unrestricted use, distribution, and reproduction in any medium, provided the original author and source are credited. 
Citation: Smaropoulos E, Papakrivou E, Netskos D, Gkikas O (2017) Open Amputation in Preterm Neonatal Digits With Post-Operative Management Using Medical Grade Honey. Int J Pediatr Neonat Care 3: 125. doi: https://doi.org/10.15344/2455-2364/2017/125

Page 2 of 3

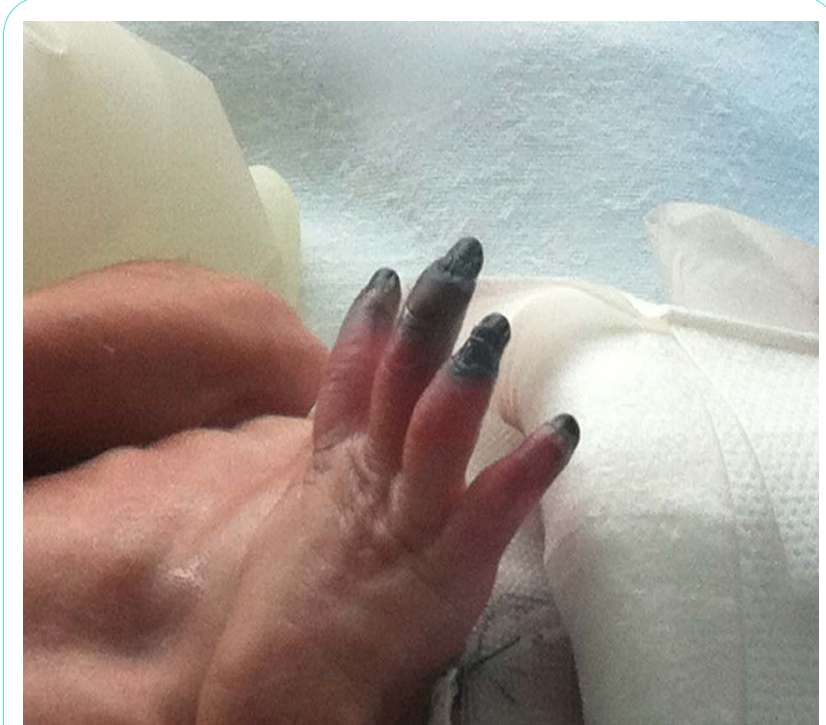

Figure 1: Dry gangrene presented 3 days after extravazation injury.

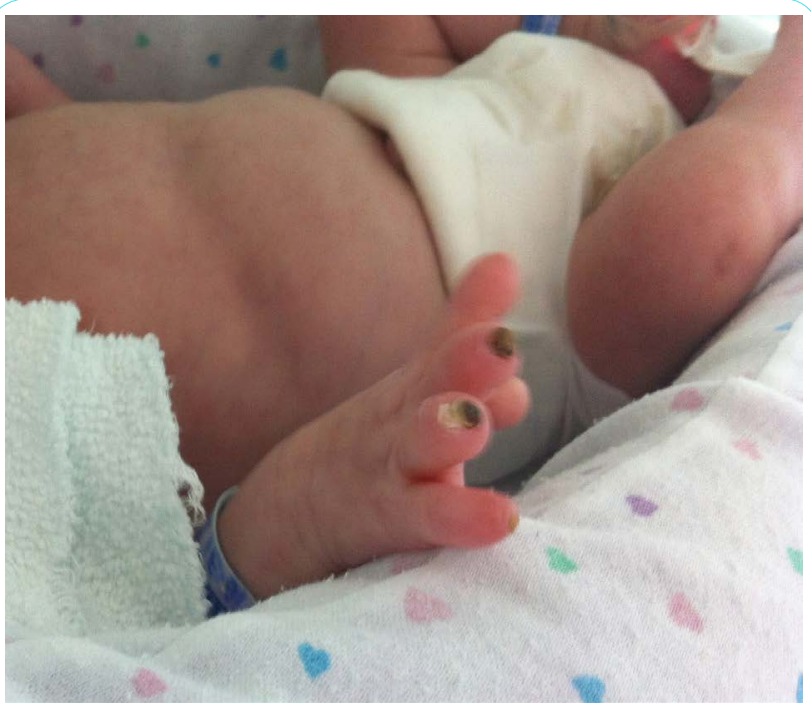

Figure 2: Post-amputation, performed at 8 days of life.

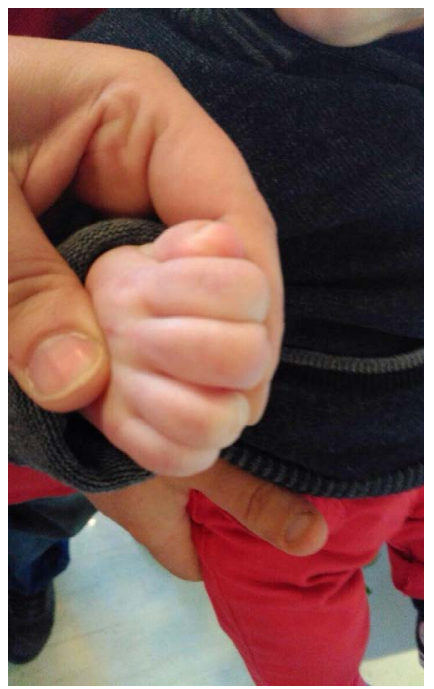

Figure 3: Follow-up at 7 months, fully healed. a full-term baby $[7,8]$. This deficiency in SC function leads to reduced water retention in the newborn skin, leaving the first line of defense of the innate immune system impaired $[2,7,8]$. Because there are so few layers of skin, even superficial wounds in neonates may be considered full thickness due to the under developed skin condition and damage incurred [9]. Additionally, at less than 28 weeks of gestation there is no vernix caseosa covering, which is made up mainly of water, proteins and lipids and which serves biomechanical and waterbinding purposes $[2,8]$. The incompetent epidermal barrier often predisposes neonates to the development of sepsis or other severe infections. Therefore, when dealing with wounds in such fragile skin, use of adhesives, for example, and respective removal requires particular care to avoid epidermal stripping [2].

Not only are neonates at higher risk of suffering extravazation injuries due to various factors, but they also lack the ability to localize and/or report symptoms related to such events. Their poor venous integrity and decreased peripheral circulation, in combination with the frequent need for longer durations of therapy all translate into a challenge in NICUs [10].

Gamma sterilized medical grade honey, possesses intrinsic bioactivities and physical properties that make it ideal for wound healing and post-operative use. Among its unique characteristics, it demonstrates broad-spectrum antibacterial activity due to hydrogen peroxide, promotes autolytic debridement and creates a favorable environment for the wound bed $[7,9]$. Though raw and commercial or culinary honey use has been documented around the world, it should not be employed in wound care due to lack of complete sterilization [7].

\section{Conclusion}

This case study contributes towards expanding existing knowledge regarding medical grade honey use in wound care, particularly in the world of pediatrics and neonatology, which still lacks data. Its safe application and absence of adverse effects in this instance addresses potential concerns regarding its use in neonates and infants, such as toxicity and botulism. Because of the frequency with which skin injuries occur in NICUs it is important to develop new and efficient tools for treating such fragile populations. Continued research is required in order to incorporate medical grade honey products into standardized wound protocols and to reach consensus-based guidelines.

\section{Competing Interests}

The authors declare that they have no competing interests.

\section{References}

1. Pammi M, O'Brien JL, Ajami NJ, Wong MC, Versalovic J, et al. (2017) Development of the cutaneous microbiome in the preterm infant: A prospective longitudinal study. PLoS ONE 12: e0176669.

2. Oranges T, Dini V, Romanelli M (2015) Skin physiology of the neonate and infant: Clinical implications. Advances in Wound Care 4: 587-595.

3. Wilkins CE, Emmerson AJB (2004) Extravasation injuries on regional neonatal units. Arch Dis Child Fetal Neonatal Ed 89: F273-F275.

4. Kostogloudis N, Demiri E, Tsimponis A, Dionyssiou D, lonnidis S, et al. (2015) Severe Extravasation Injuries in Neonates: A Report of 34 Cases. Pediatric Dermatology 32: 830-835.

5. Cooper R (2016) Honey for wound care in the 21st century. J of Wound Care 25: 544-552. 
Citation: Smaropoulos E, Papakrivou E, Netskos D, Gkikas O (2017) Open Amputation in Preterm Neonatal Digits With Post-Operative Management Using Medical Grade Honey. Int J Pediatr Neonat Care 3: 125. doi: https://doi.org/10.15344/2455-2364/2017/125

Page 3 of 3

6. Saikaly SK, Khachemoune A (2017) Honey and wound healing: An update. Am J Clin Dermatol 18: 237-251.

7. Mohr LD, Reyna R, Amaya R (2014) Neonatal case studies using active Leptospermum honey. J Wound Ostomy Continence Nurs 41: 213-218.

8. Visscher MO, Adam R, Brink S, Odio M (2014) Newborn infant skin: Physiology, development and care. Clin Dermatol 33: 271-280.

9. Schierholz E (2017) Leptospermum honey for wound care in an extremely premature infant. Adv Neonatal Care 17: 27-32.

10. Hirsch SD, Powers JM, Rhodes JL (2017) Neonatal Soft Tissue Reconstruction Using a Bioengineered Skin Substitute. J Craniofac Surg 28: 489-491. 